International Joumal of Biological Sciences
2010; 6(3):225-227

(c) Ivyspring International Publisher. All rights reserved

Letter

\title{
Leukocyte infiltration as a surrogate marker for diagnosis of invasion
}

\author{
Aijun Liu, Lixin Wei ${ }^{\bowtie}$ \\ Department of Pathology, Chinese PLA General Hospital, 28 Fuxing Rd., Beijing 100853, P.R. China
}

$\checkmark$ Corresponding author: Professor Lixin Wei, weilx301@263.net

Received: 2010.03.24; Accepted: 2010.04.27; Published: 2010.04.28

\begin{abstract}
Dr. YG Man's hypothesis that breast tumor invasion is triggered by the aberrant leukocyte infiltration induced by degeneration of myoepithelial cells holds a lot of truth in our clinical practice, and leukocyte infiltration may be regarded as a surrogate marker for diagnosis of invasion.
\end{abstract}

Key words: Leukocyte infiltration, tumor invasion, pathological diagnosis

We read with interest the article "Aberrant leukocyte infiltration: a direct trigger for breast tumor invasion and metastasis" by Man YG [1]. The author proposed a novel hypothesis that breast tumor invasion is triggered by the aberrant leukocyte infiltration induced by degeneration of myoepithelial cells, which, in turn, leads to the focal disruptions of myoepithelial layer. The author presented here a unique mechanism that leukocyte infiltration is a triggering factor for tumor metastasis, which could potentially be applied to all epithelium-derived tumors, as leukocytes are widely distributed in abundance in almost all human tissues.

Dr. Man's hypothesis indeed holds a lot of truth in our clinical practice. We have found that leukocytes, behaving like obligated partners to the tumor cells, infiltrate around and/or in the invasive foci of carcinomas. Among the infiltrating leukocytes, lymphocytes are the most commonly observed, followed by neutrophils, plasmocytes, macrophages and even eosinophils. This phenomenon has raised increasing attentions and has been investigated in other studies [3-6].

It is widely accepted that immunological competence plays an important role in tumor progression. Herein, we do not intend to discuss the underlying molecular mechanism of leukocyte infiltration. What concerns us the most in clinical practice is its potential application in assisting surgical pathologist to make an early diagnosis of a certain tumor. We would like to take the microinvasive squamous cell carcinoma of the uterine cervix for an instance.

As the treatment strategies and prognosis for pre-invasive lesion and microinvasive squamous carcinoma of uterine cervix are quite different, detection of areas of minimal invasion is of critical importance. However, the distinction between non-invasion and microinvasion is often challenging for surgical pathologists, especially in very small specimens. It is well known that cancer invasion is the result of cancer cell disruption of the epithelial basement membrane followed by migration into the stroma. We found that, in the foci of microinvasion, the basement membrane is attenuated and blurred by leukocyte infiltration, suggesting that leukocytes was associated with early invasion in cervical squamous neoplasms (Figure 1). This may be consistent with Dr. Man's notion that cancer associated leukocyte infiltration implicates the initiation of tumor dissemination or the transition of tumor from in situ to invasive. However, it could also be possible that leukocyte infiltration is the response to cancer invasion $[5,6]$. No matter the leukocyte infiltration is a cause or a result of cancer invasion; we could regard it as a surrogate marker for diagnosis of microinvasion. Nevertheless, the presence of leukocytes in the inflammatory lesions of cervix should be 
differentiated from those near invasive foci. These associations should be excluded when evaluating the significance of leukocytes infiltration in squamous neoplastic lesions of the cervix.

As it is known that HPV DNA can be detected in most, if not virtually all, cervical cancers. However,
HPV infection, on one hand, is not believed to be inflammatory, which means HPV per se cannot cause leukocyte infiltration. On the other hand, there is some epidemiological evidence suggesting that inflammation might be linked to cervical cancers, perhaps as a HPV co-factor [7].
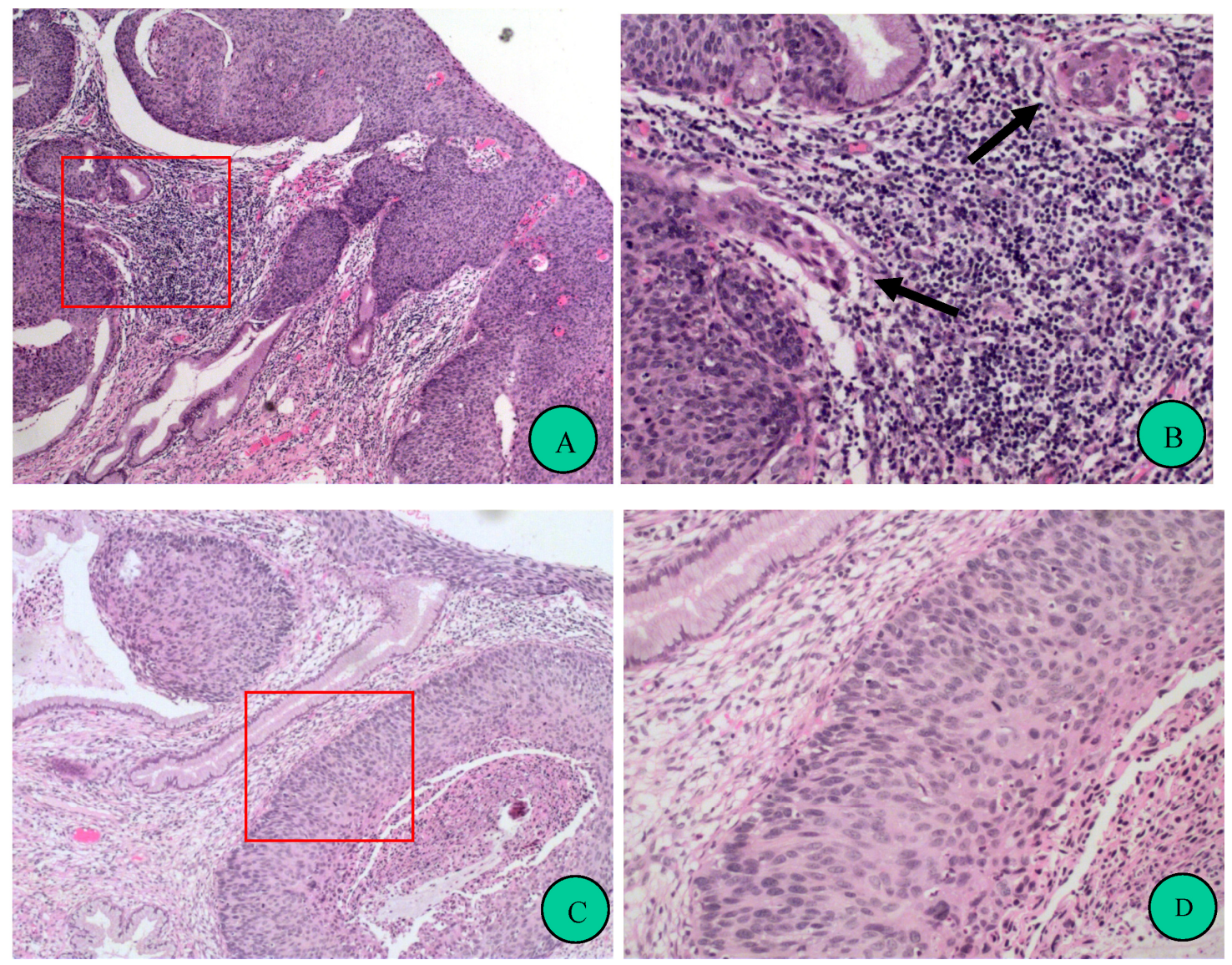

Figure I. Prominent leukocyte infiltration is frequently found in microinvasive foci of cervical squamous neoplasms. A, Representative case of cervical intraepithelial neoplasia, grade 3 (CIN 3) with microinvasion (hematoxylin-eosin stain, original magnification 100x). Red frame indicates an area magnified in B. B, Focal magnification of A. Arrows indicate microinvasive foci, around which are leucocytes infiltrations, resulting in blur of the border of the epithelial nest. Twenty-six cases of CIN3 with microinvasion were analyzed and all of them showed prominent leukocyte infiltrations in the microinvasive foci. C, Representative case of CIN 3 without microinvasion (hematoxylin-eosin stain, original magnification I00x). Red frame indicates an area magnified in D. D, Focal magnification of C. Compared to B, much less leukocyte infiltration presents in the stroma, and the border of the gland involved by CIN is "clean". Thirty-nine cases of CIN2-3 were observed. Most (79.5\%) of them showed few leukocyte infiltrations, and other cases had more leukocyte infiltrations around both normal and CIN-involved glands.

\section{Conflict of Interest}

The authors have declared that no conflict of interest exists.

\section{References}

1. Man YG. Aberrant leukocyte infiltration: a direct trigger for breast tumor invasion and metastasis. Int J Biol Sci. 2010; 6:129-32.
2. Man YG. Focal degeneration of aged or injured myoepithelial cells and the resultant auto-immunoreactions are trigger factors for breast tumor invasion. Med Hypotheses. 2007; 69: 1340-57.

3. Spiegel GW, Ashraf M, and Brooks JJS. Eosinophils as a Marker for Invasion in Cervical Squamous Neoplastic Lesions. Int J Gynecol Pathol, 2002; 21:117-24.

4. Woo YL, Sterling J, Damay I, et al. Characterising the local immune responses in cervical intraepithelial neoplasia: a cross-sectional and longitudinal analysis. BJOG. 2008; 115: 1616-21. 
5. Schottenfeld D and Beebe-Dimmer J. Chronic Inflammation: A Common and Important Factor in the Pathogenesis of Neoplasia. CA Cancer J Clin. 2006; 56: 69-83.

6. Mantovani A, Sica A. Macrophages, innate immunity and cancer: balance, tolerance, and diversity. Curr Opin Immunol. 2010; 22: 231-7.

7. Castle PE, Hillier SL, Rabe LK, et al. An Association of Cervical Inflammation with High-Grade Cervical Neoplasia in Women Infected with Oncogenic Human Papillomavirus. Cancer Epidem Biomar. 2001; 10: 1021-7. 